\title{
Feeding Infants and Toddlers-An Intuitive and Simple Action, Is It?
}

\author{
Pnina Hertz \\ Hadassah Medical Center, Jerusalem, Israel
}

\begin{abstract}
Feeding infants and toddlers is a natural action that can at times be challenging. A chronic illness or a neurodevelopmental problem maybe the source of the initial difficulty. When feeding is also difficult for the parent, an additional component is present which may exacerbate the situation and require therapeutic intervention, including diagnosis and parental counseling. Integrative therapy is essential in the processes of diagnosis and treatment of infants and toddlers and parents with eating and feeding challenges. This is presented in the paper in four cases from the Feeding and Eating Clinic for infants and toddlers.
\end{abstract}

Keywords: eating, parental feeding, treatment of infants and toddlers, integrative therapy

\section{Introduction}

Infants and toddlers' eating disorders are one of the most common problems about which parents advise at the Feeding and Eating Clinic for infants and toddlers. Eating disorders among young children are evident in overeating, undereating, or in eating with unique and unusual characteristics (Zero to Three, 2016). Children brought to the clinic usually suffer from undereating or failure to thrive. The first common signs are low weight, possible weight loss, and poor nutrition status. Additional signs sometimes appeared later, are not necessarily physical and may have long-term implications. For therapists in the mental health field, these signs are our primary interest and focus.

In infancy, when the helpless baby is dependent on external sources for nursing and provision, eating and feeding are intertwined. We are working with a dyad, with each side contributing its share to the experience of eating and feeding.

Feeding challenges in a specific point of time are sometimes impacted by the past. A significant part of the known population arrived in this world in a manner that was not necessarily soft, benevolent, anticipated, or rewarding for the parental experience. This can provide fertile ground for potential parental anxiety, which usually tends to be maternal anxiety. In more complex cases, when it is hard to discern the more benevolent parent, we diagnose and treat parenting that is potentially damaging because of primary pathology, and intergenerational transmission of eating disorders or more extensive psychosocial complexity (Fraiberg, Adelson, \& Shapiro, 1975). Between the two extremes-the parent who is mobilized but having a hard time and the parent likely to harm the child, it is a wide range of cases, events, people and childrearing.

Pnina Hertz, Ph.D., Pediatric Psychologist, Hadassah Medical Center, Jerusalem, Israel. 


\section{Work Principles in an Integrative Team}

Infants and toddlers are referred by the community to the Feeding and Eating Clinic, primarily by pediatricians concerned about the baby's weight or following hospitalization in the children's ward. Few parents initiate a visit without an active or mediating referral. Parental concern focuses on weight and sometimes, nutritional status or concern about the influence on the emotional state or development. A comprehensive response to parental concerns and actual diagnoses requires a holistic approach, which explores individually every aspect influencing the eating disorder and recommends an integrative therapeutic approach.

The initial meeting with the family also reveals parental characteristics and perspectives regarding eating, raising, and even educating children. This familiarity facilitates individual treatment based on the fundamental principles of the therapeutic team. On the one hand, there are children with complex issues, usually with a medical background and secondary eating and feeding challenges. On the other hand, there are toddlers and even infants who have developed a problem, may resist or refuse food, and be restless at mealtime, without an apparent reason or background. The reasons are clarified in confronting the psychological and family issues.

With most infants and toddlers, connections between medical and primary functional factors that influence eating ability and psychological and environmental factors influence positions regarding food and the feeder, which "feed" on each other. The multidimensionality of the disorder requires a diagnostic and integrative approach; consequently, the team is made up of medical and mental health professionals and a nutritionist. These professionals are fully committed to collaborating, considering environmental aspects, and following a joint diagnostic process, and focus on the appropriate treatment.

The staff treats illnesses, such as metabolic illnesses, which are also expressed in eating challenges and the need for appropriate support and planning, follow growth measurements compared with same-age children as well as specific syndromes, and even recommend meal preparation with the proper nutrition. Instructions are given as to how to feed with feeding strategies, appropriate guidelines for the given developmental stage, and when necessary, professional connections with the community on topics, such as adapting equipment, day-to-day support in the community for especially challenged families and finding the right educational frameworks. A significant number of the infants and toddlers treated in the clinics are integrated in rehab day centers.

Working with the parents also focuses on their emotional state, expectations vis-à-vis possible disappointments, difficulty in accepting complex situations, ongoing psychological support on the personal and parental level, and connection with the community.

\section{Family Situations and Personal Characteristics Connected With Non-beneficial Feeding}

Parents of infants, primarily females, are anxious regarding their weight in a specific period. This involvement includes the development potential and long-term influences of eating disorders on adolescence.

Negative food associations may arise when the infant's environment applies pressure regarding expected food amounts and variety. When pressure and expectations become heightened around food, mealtime can become "a battleground" for the parent in approach and interpretation. The parent is not always aware of his/her part in the deteriorating situation and in shaping the baby's negative relationship to food and food times. The appropriate therapeutic work can clarify to the parent that it takes two to tango, just as it takes two to fight. Samish-Talor (2006) presented factors that influence the dyadic connection in eating that disrupt processes, 
including mistakes in timing, length of meals, over-stimulation, or changing the baby's routine before he/she is ready.

Prior to these insights, meals will often be tense, characterized by a fear of failure and the pessimistic parent's thinking that the challenging meals illustrate parental inability to provide the baby's basis needs. This parental experience connects paradoxically with parental difficulty in letting the baby separate and progress in acquiring eating skills (Winnicott, 1971).

The child who refuses to eat is likely to be perceived and perceive himself/herself as problematic within the family. When parents allow an open discussion on "negative" behavior, such as the tendency to refuse or be stubborn, the child's difference is underlined in the siblings' eyes. This not necessarily conscious parental tendency sometimes hurts the whole family, and not only the food refuser.

This approach fosters coalitions and asymmetric relations within the family. Ongoing putdowns of one family member in the eyes of the others can create the new role of "the identified patient", who functions as the family sacrifice (Menuchin, 1982).

Put-downs or negative parenting in the family environment is also seen in the clinic with parents who cannot come to their children's defense or identify their strengths and abilities. Negative positions tend to harm the development of the parental ability to enthuse about the child, and consequently, the child's ability for healthy emotional development decreases. A parent who needs control due to his/her own anxiety can block the child's opportunities for autonomy and foster dependency, in feeding situations as well.

In extreme states, such as Munchausen by Proxy (Psychiatric Manual) (American Psychiatric Association, 2013), damaging gaps in perception that are not always bridgeable can be identified. The mechanism behind this basic failure in essential roles is very complicated and requires its own discussion to supervise and prevent danger to a helpless population.

\section{Expressions of Non-optimal Feeding}

A lack of basic foods is infrequent but exists in socio-economically weak groups, who do not have easy access to relevant childrearing information. Possible expressions can be choosing food texture or nutritional combination unsuitable to the child's age. Formula may be diluted to save money or prepared with more than the recommended amount due to mistaken judgment.

The nursing infant's feeding is usually based on maternal intuition and the correct analysis of the integrative ability of breathing, sucking, and swallowing. An intuitive parent will adapt the feeding according to the developed functioning of the baby's mouth. She will also check if the infant can open his/her mouth for the content of the spoon moving in his/her direction and will wait for an expression of interest. A consistently dreamy or distracted parent may not identify signs of hunger or satiety and may feed too slowly or too quickly. A parent who is anxious because of the baby's perceived small size may speed up the feeding pace or cause specific discomfort that may develop into resistance following cumulative non-enjoyable feeding experiences.

A parent who has difficulty facilitating the infant's emotional development or other growth and even prevents achievements may also express this in the area of eating. Some parents place the infant in an unsuitable position for their motor development or in a chair not adjusted to the child's need for independence and control during meals. A parent unaware of the child's developmental needs may serve food with the texture or portion size appropriate for a younger child.

Food temperature is important and not necessarily identical for all same age infants as this depends on the 
infant's sensory regulation. Attuned parenting is important to identify preferences and other sensory nuances for a positive eating experience (Samish-Talor, 2006).

Feeding accompanied by ongoing noise or bright lights can be unpleasant and pressured instead of tranquil. We see this tendency in stressed-out parents who accompany meals with a lot of talking. In treatment, the importance of moderation is explained.

The short case studies presented below are examples of non-optimal eating, with different levels of difficulty and a range of parental and family profiles, supportive or challenging, in the nuclear family and wider circles.

\section{Four Cases}

\section{Case A: The David Family}

The David family with parents and a one-year-old baby came to the Feeding Clinic that offers an integrative team of doctors, a psychologist, and a nutritionist. The family broadcast love and friendship and sharing as well as the couple's mutual respect. This triad also had the benefit of the extended family's support, good primary experiences of parenting, and family examples of closeness and sensitivity. Gadi, the David's firstborn son, was born early. His preemie status and infant hospitalizations shook the parents' confidence and caused breaks in the natural processes of acquiring parental abilities. His infancy was characterized by parental anxiety and worry as to his medical, nutritional, and developmental future. The first experience left its mark. When the staff met the family, the past was not blurred and was experienced as the ongoing present. The future threatened with questions and doubts as to the infant's health and development. When Gadi's eating abilities were encouraging, his mother, the primary escort to the clinic, had difficulty seeing the visible signs in front of her. This difficulty stemmed from over involvement with the past and exhausting repetitive thoughts regarding the future that made her miss the amazement and pleasure in her son's achievements and accomplishments. With time and sensitive treatment, the mother could intellectually and even emotionally access this missed opportunity and with these insights connect to the here and now experiences, seeing the child progress towards a good future despite his preemie past.

This case describes parental vulnerability due to life's exigencies. The vulnerability is mediated thanks to the parent's initial potential abilities that gradually come to the fore, following a process of intervention and thanks as well to several circles of support.

\section{Case B: Ella and Gabriella}

Ella came to the clinic with Gabriella following Gabriella's hospitalization in the children's ward. Ella had shared her concerns about her daughter's eating and sleepiness and therefore Gabriella underwent an extensive range of tests whose results were normal. There was no special obstetric issue. The family situation, including the partner's involvement, was wrapped in secrecy. The mother's information was not overly coherent, and her manner of reporting and affect added to the feeling of her unreliability.

Despite a normal infancy, the mother's concerns for the infant's thriving and food sensitivity developed. Sensitivity to the formulas was checked and not automatically ruled out but the medical staff related less to this aspect than did the mother. The sense that Ella could worsen Gabriella's situation aroused concern among the staff. Over time, Ella developed a splitting defense mechanism towards the staff; beneficial staff were those who validated her concerns while the rest were non-beneficial because they did not see an objective medical 
problem. Excessive treatments were discovered, including surgical intervention initiated by Ella, as well as her moving around to other doctors and treatment centers. Concerns as to Ella's possible harming Gabriella required a turn to the authorities and ongoing follow-up, especially as Ella ignored the treatment we had recommended.

The inaccessibility of the mother's past and the parenting she had experienced, heightened the "Ghosts in the Nursery" feeling (Fraiberg et al., 1975). No therapeutic treatment was established, and important primary topics potentially harmful to the helpless infant were not explored. We had to come to terms with these limits and hope that the appropriate authorities would take action.

\section{Case C: Chen}

Rivka was separated from her partner when she came in to the clinic with her one-year-old daughter Chen. Chen was hospitalized in the children's ward for low weight due to a fever and then transferred for treatment to the integrative Feeding Clinic who would provide guidelines for Rivka and treat the feeding issues, failure to thrive and vomiting. As an infant, Chen had a heightened vomiting reflex, a common indicator of infant anorexia (Levine et al., 2001). The staff's impression, after an integrated intake that crosschecked medical, nutritional, and psychological data was that Chen would physical grow when Rivka successfully released rigid feeding patterns and better identified Chen's hunger and satiety signals and communication initiatives. The initial hospitalization occurred at the most chaotic period in the household, including the breakup in the couple's relationship. Chen became "the identified patient"; the family pathology and the couple's breakup drained onto her.

Chen showed interest in and a positive approach to food and especially regarding the mother's interpretation. We did not see resistance to food but rather a desire for a multi-sensory experience and pleasure from the increasing exposure to taste and textures. The developed and developing toddler had to wait for her mother to develop. When Rivka made the decisions right for her regarding her partner, she released Chen from the role of patient and could see her as a healthy and well-developed child who brought pleasure. Everything occurred gradually but the ability to change and relinquish rigid behavior was promising.

When parental ability for change is limited or slower than the infant's potential for change in feeding patterns and calorie intake, validation of the parents' difficulty and factors impeding change is effective for parents with an uncomplicated past and especially for those who had well-enough parenting.

\section{Case D: Alma and Her Parents}

Alma was 10 months old, looked thin and weak, when referred by her concerned pediatrician to the Feeding Clinic. Her development was also delayed. The reported family background made us suspect high-risk intergenerational difficulties of feeding and containment. Alma's parents shared the same positions and arguments, but their alliance was against the health and welfare systems involved in their supervision. Their matched anti-position worsened things, including the baby's low weight and their inability to resolve the feeding disorder and delayed development.

Parental supervision was necessary because of the baby's serious failure to thrive and the parents' inconsistent following of medical recommendations. Alma also came to the clinic because of the parents' tendency to move between diagnostic frameworks and therapists. These transitions and overuse of approaches and techniques resulted in inconsistent treatment and problems with perseverance. The parents had a hard time committing to one main approach and to consistency. They even garbled clearly defined medical instructions 
and appeared to not understand, despite their apparent cognition.

Alma was fed smaller amounts than those recommended and required. She clearly wanted to be fed more quickly. In the advanced stages of therapy and after her medical situation was stabilized, the work included "confrontations "on the non-benevolent feeding practices. Clearly, a long process would be needed for entanglements around food, internalized parenting and changing non-benevolent parental influences into benevolent parental influences (Liberman, Pardon, Van Horn, \& Harris, 2005).

\section{Discussion}

The population that comes to the Feeding Clinics in Israel is culturally diverse and varied in its perspectives on feeding and the place of food in religious, social, and even educational contexts.

The medical background prior to the food disorder can also be different, a case in point, infancy anorexia. Undereating in infants is of concern to all parents because of the immediate challenges and the long-term implications for the infant's emotional, developmental, social, and educational future. There are shared patterns in the population that come for treatment, regardless of gender and socio-economic background.

The available parent at his best is characterized by precise descriptions. He can enjoy the child, treat him/her with equanimity and appreciate therapeutic achievements. He gives the required amounts of food and does not deviate from therapeutic recommendations. He initiates but does not contradict professional recommendations. The parent's concerns and anxieties tend to make him/her vulnerable. The vulnerability releases patterns that challenge the staff, some of which are described below.

Parental narcissism is a driving force for the parent's defense of the child. When the therapist is forced to take on this role vis-à-vis the blaming parent, the child is probably not experiencing the parent's pleasure and delight, which are vital for mental hygiene. It is worthwhile checking if the child is missing a parental experience highlighting strengths. A general negative bias regarding the child's situation or behavior raises the need to check what happens when parents cannot see the positive reality or identify welcome developments because of successful therapy. We are concerned when the observed infant or toddler is significantly different from the parents' report. A parent who diminishes the actual calorie intake or variety of food eaten is a common example of this behavior.

We need the right dose of parental involvement and collaboration, suited to the child's needs and stage of therapy. Too frequent clinic visits mean that energy is expended in the wrong places. Sometimes, both parents' involvement needs clarification that may not necessarily be measured in the number of sessions attended. A parent's ongoing absence may indicate resistance to therapy and therefore necessitate a shift in the therapeutic approach, including requests for a one-on-one meeting and individual discussions to clarify difficulties. Covert resistance sometimes hints at distress - of the couple or other distress and requires comprehensive therapy.

Adequate treatment of food disorders depends on the staff's accessibility to the relevant personal and familial information. The more open and direct the communication, the more therapy can be adjusted to the child's needs, taking into account the environment and community. When the gap between the overt and covert messages shrinks, the ability to offer effective and significant therapy for the decided-upon goals increases.

When things are shrouded in secrecy, the challenge is greater. Disclosure of the secret may happen with time and efforts to understand the secret's place and function in the life of the family. When communication is laden with examples, the clinician may, at the end of the session, feel overwhelmed with the exhaustive details and pieces of information that are not a coherent statement. This confusion makes therapeutic decisions more 
difficult. Confusing or contradictory information may indicate the parents' ambivalence to therapy or differences within the family. A typical Israeli family includes involved grandparents who also influence parental beliefs and perceptions regarding eating, feeding, and containment.

These examples, known in the community and in the pediatrician's office, are a red light signal for families needing therapy in the Feeding and Eating Clinics for infants and toddlers. Intervention invites renewed thinking and facilitates a planned referral to the clinic for integrative therapy. Holistic treatment at critical stages of health cognitive and emotional development is advantageous and important for the individual and society.

\section{References}

American Psychiatric Association. (2013). Diagnostic and statistical manual of mental disorders (5th ed.). Arlington, VA: American Psychiatric Association.

Ammaniti, M., Lucarelli, L., Climino, S., D’Olimpio, F., \& Chattor, I. (2012). Feeding disorders of infancy: A longitudinal study to middle childhood. International Journal of Eating Disorders, 45(2), 272-280.

Fraiberg, S., Adelson, E., \& Shapiro, V. (1975). Ghosts in the nursery: A psychoanalytic approach to the problems of impaired infant-mother relationships. Journal of the American Academy of Child and Adolescent Psychiatry, 14(3), 387-421.

Levine, A., Bachar, L., Zangen, T., Mizrachi, A., Levy, A., Dalal, I., ... Boaz, M. (2011). Screening criteria for diagnosis of infantile feeding disorders as a cause of poor feeding and food refusal. Journal of Pediatric Gastroenterology and Nutrition, 52(5), 563-568.

Liberman, A. F., Pardon, E., Van Horn, P., \& Harris, W. W. (2005). Angels in the nursery: The intergenerational transmission of benevolent parental influences. Infant Mental Health Journal, 26(6), 504-520.

Menuchin, S. (1982). Mishpacha veterapia mishpachtit (Family and family therapy). Modan: Ben Shemen.

Samish-Talor, S. (2006). Aruchot yeladim kehavayot meazvot hitpathut (Children's meals as development-shaping experiences). Ah: Kiryat Bialik.

Sega, I., Tirosh, A., Sinai, T., Alony, S., Korenfeld, L., Zangen, T., ... Levine, A. (2014). Role reversal method for treatment of food refusal associated with infantile feeding disorders. JPGN, 58, 739-742.

Winnicott, D. (1971). Mizhak umeziot (Playing and reality). Oved: Tel Aviv.

Zero to Three. (2016). DC:0-5: Diagnostic classification of mental health and developmental health and developmental disorders of infancy and early childhood. Washington, DC: Author. 